\title{
TUBERCULOSIS MIMICKING ABDOMINAL MALIGNANCY IN IMMUNOSUPPRESSED PATIENT WITH SARCOIDOSIS
}

Guilherme Pereira Carlesso ${ }^{1, \star}$, Heloísa Rodrigues Silva Catalá ${ }^{1}$, Andressa Higa Shinzato ${ }^{1}$, Isadora Medina ${ }^{1}$, Marcela Miguens Castelar Pinheiro ${ }^{1}$, Felipe de Lacerda Veiga ${ }^{1}$, Stella Falcadi Vendramine ${ }^{1}$, Luíza Sá e Rêgo Tupinambá ${ }^{1}$, Pedro Matos ${ }^{1}$, Edgard Torres dos Reis Neto ${ }^{1}$

1.Universidade Federal de São Paulo, São Paulo (SP), Brazil.

*Corresponding author: guilherme.p.carlesso@gmail.com

\section{BACKGROUND}

Abdominal tuberculosis (TB) includes gastrointestinal, peritoneal and lymph nodal involvement, being responsible for about $5 \%$ of TB cases in the world. In its turn, sarcoidosis is a systemic chronic granulomatous disease characterized by non-necrotizing granulomas hat also can affect gastrointestinal organs. Peritoneal involvement by neoplasms, TB and sarcoidosis continuous to be a challenging diagnosis in immunosuppressed patients, particularly because its clinical and radiographic similarities.

\section{CASE REPORT}

A 39-year-old male was diagnosed with sarcoidosis in 2016 when presented erythema nodosum, pulmonary lymph nodes and heart disease (conduction disorder with pacemaker implant and heart failure). The disease was considered in remission with mycophenolate mofetil $2 \mathrm{~g} /$ day and prednisone $5 \mathrm{mg} /$ day since 2017. In the 15 days prior to hospitalization, he started to present daily night fever $\left(40{ }^{\circ} \mathrm{C}\right)$ associated with nausea, vomiting, diffuse abdominal pain, liquid diarrhea and weight loss of $7 \mathrm{~kg}$ without improvement with ciprofloxacin. Laboratory tests showed leukocytosis $\left(22.820 / \mathrm{mm}^{3}\right)$, lymphopenia, as well as elevated C reactive protein $(430 \mathrm{mg} / \mathrm{L})$, liver transaminases and canalicular enzymes (AST $87 \mathrm{U} / \mathrm{L}$, ALT $81 \mathrm{U} / \mathrm{L}$, FA $307 \mathrm{U} / \mathrm{L}$ and GGT 455 U/L). Computerized tomography (CT) demonstrated periampullary tumor with multiples peritoneal implants forming "omental cake" aspect (Fig. 1), which were biopsied by interventional radiology. After a few days, the patient developed sudden dyspnea and a new CT scan identified bilateral pleural effusion classified as lymphomononuclear exudate after thoracentesis. Sputum staining of acid-fast bacilli (AFB) and rapid molecular test (RMT) GeneXpert were negative and inconclusive, respectively. Rapid molecular test in sputum was repeated and a rifampicin-resistant Mycobacterium tuberculosis was detected. Omental implant biopsy exhibited granulomatous inflammatory chronic process with AFB. Rifampicin, isoniazid, pyrazinamide, ethambutol associated with levofloxacin was prescribed evolving with resolution of symptoms.

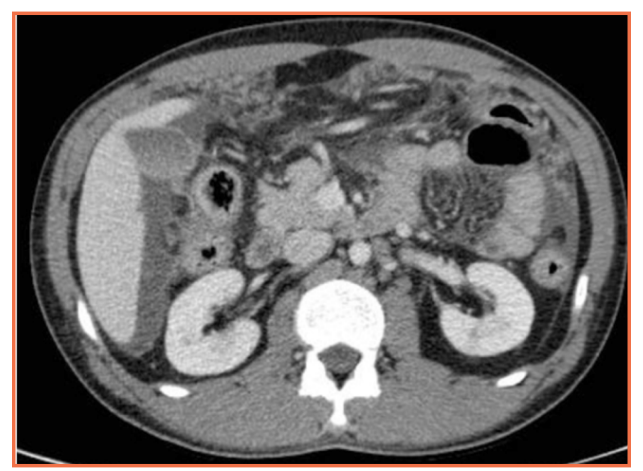

Figure 1. Computerized tomography (CT) demonstrated periampullary tumor with multiple peritoneal implants forming "omental cake" aspect.

\section{CONCLUSION}

The differential diagnosis among abdominal TB, intestinal metastatic neoplasm and sarcoidosis activity is a challenge in such cases, mainly due to omental cake appearance in image exam. As no radiographic exam is a sufficient tool to perform the differential diagnosis, anatomopathological study is the gold-standard to investigate and confirm abdominal TB. 